\title{
Fichte und Reimer.
}

Mit einem unveröffentlichten Brief Fichtes.

Von Adolfo Rava.

Es ist mir eine besondere Freude, noch in diesem Jubiläumsjahr einige unveröffentlichte, in meinem Besitz befindliche Zeilen von Fichtes Hand, durch die auch um Fichtes Andenken so sehr verdienten Kantstudien bekanntzugeben. Die kurze Zuschrift ist auf ein Blatt im Format $19 \times 23 \mathrm{~cm}$. geschrieben, das noch die Spuren der damals üblichen Faltung zeigt und auf der Adressseite ein kleines Siegel trägt. Sie lautet:

„Ich habe, mein theurer Freund, keinen Messkata$\log$ gesehen, und befinde mich daher über manches in ignorantia, nebme daher Zuflucht zu Ihnen.

Ist nicht ein Büchlein von einem Dr. Rückert (vielleicht heisst es Realismus) erschienen? Haben Sie doch die Güte, mir es baldmöglichst zu verschaffen.

Sollte es sich in Berlin gar nicht befinden, so bitte ich Sie, es aus Leipzig zu verschreiben.

(Adresse)

Herrn Buchhändler Reimer von der Real Schulbuchhandlung

\section{allhier}

Der Ibrige

Fichte.

Das Buch, welches sich Fichte vermittelst dieses Briefes verschaffen wollte, hiess eigentlich: Joseph Rückert, Der Realismus, oder Grundsätze zu einer durchaus prakiischen Philosophie, Leipzig, Göschen, 1801. Da es entweder zur Neujahrs- oder zur Ostermesse erschienen ist, muss der Brief in der

Kantstudien XIX. 
ersten Hälfte des Jahres 1801 geschrieben worden sein, und zwar in Berlin, wo sich Fichte damals anfhielt. ${ }^{1}$ )

In diesem Buch nimmt der. Verfasser wohl Stellung gegen Fichte, ohne sich jedoch dessen Einfluss entziehen zu wollen. Offeubar inatte Fichte nun irgenwie Nachricht von der neuen Veröffentlichung erhalten, die er daher sehr begierig war kennen zu lernen, was man leicht begreifen wird, wenn man an die vielen Angriffe denkt, welchen er zu einer Zeit ausgesetzt war, wo er sich sogar von Manchem, der ihm vorher philosophisch nahe gestanden, verlassen sehen musste.

Nach dem Bericht von Fichtes Sohn hatte Rückert selbst seine Schrift an Fichte gesendet, und ihn zur Prüfung derselben aufgefordert. ${ }^{2}$ ) Das ist durch unser Billet keinesfalls ausgeschlossen, da es ganz gut möglich ist, dass Fichte das Buch vom Verfasser erhielt, während er es schon bei seinem Buchhändler bestellt hatte. Sicher ist jedenfalls, dass Fichte dem Verfasser einige Bemerkungen über sein Buch zukommen liess, was einen Briefwechsel zur Folge hatte, der uns erhalten geblieben ist. ${ }^{8}$ ) Aus diesem erhellt einerseits, dass Fichte das Buch wohl strenge, aber nicht ohne Wohlwollen und nicht ohne Hoffnung in des Verfassers philosophische Zukunft beurteilte, andererseits, dass Rückert diese Kritik Fichtes, den er Freund und Lehrer nennt, mit Dankbarkeit aufnahn und in so ernste Erwägung zog, dass er den Vorsatz aussprach, den Gegenstand nochmals und in erschöpfender Weise zu behandeln. ${ }^{4}$ )

Die Unparteilichkeit und das Wohlwollen dieser Kritik (deren gute Wirkung infolgedessen nicht ausblieb) sind um so anerkennenswerter, weun man Fichtes damaligen Seelenzustand bedenkt, und

1) Im Herbst war das Buch Fichte schon bekannt (s. folgende An. merkung). Es kann also nicht zur Michaelismesse erschienen sein. Zu welcher der andern zwei Messen es jedoch erschienen ist, konnte dio Firma Göschen, an die ich mich gewandt habe, nicht feststellen. Derselbe Verfasser veröffentlichte im gleichen Jahre unter dem Namen Karl Joseph ein zweites Buch, das den sonderbaren Titel „Weltgericht der Philosophen von Thales bis Fichte" führt.

2) S. I. H. Fichte, Fichtes Leben und literarischer Briefwechsel², Leipzig 1862, II, S. 55 1 .

3) a. a. O. S. $551-4$.

4) Tatsächlich ist uns eine spätere Schrift von ihm bekannt: Über . den Charakter aller wahren Philosophie. Ein Programm, Bamberg und Würzburg 1805. 
wenn man sie mit der unbeschreiblichen Heftigkeit vergleicht, mit welcher das Buch und sein Verfasser in einem Artikel des „Krititischen Journal der Philosophie" von Schelling und Hegel angegriffen wurden. ${ }^{1}$ )

Aber mehr noch als des verlangten Buches wegen erregt das kleine Billet unser Interesse durch den freundschaftlichen Ton, in dem es gehalten ist, und der uns eine Herzlichkeit der Beziehungen zwischen Fichte und Reimer verrät, welche von den Biographen Fichtes bisher unbeachtet geblieben zu sein scheint.

Weitere zwischen Fichte und Reimer gewechselte Schriftstücke, welchen ich, angeregt durch dieses Billet, auf die Spur gekommen bin, sowie andere da und dort verstreute Nachrichten, ermöglichen es diesen Beziehungen, die nicht ohne Bedeutung für die Geschichte des deutschen Geistes geblieben sind, wenigstens in ibren Hauptzügen nachzugehen, ${ }^{2}$ ) was ich in folgendem versuchen will.

1) Rückert und Weiss, oder Die Philosophie, zu der es keines Denkens und Wissens bedarf, in Krit. Journ. Bd. I, St. 2. Der Aufsatz ist in Schelling's Sämtlichen Werken, I. Abt., Bd. 5, abgedruckt; er gehört jedoch zu jenen, von welchen es zweifelhaft ist, ob Schelling oder Hegel der Verfasser war. Wenn es jedoch wahr ist, wie Hayu behauptet, dass „nur Schelling es verstand, die souveräne Virtuosität des Schimpfens mit allem Glanz der Diktion, mit der Miene und dem Ton der Vornehmheit zu verbinden", so ist der Aufsatz zweifelsohne von Schelling. S. Haym, Hegel und seine Zeit, Berlin, 1857, S. 157.

2) Der Urenkel Reimers, Georg Hirzel (sein Grossvater Salomon Hirzel war mit Reimers Tochter Anna verheiratet), welcher im Besitz vieler von seinem Urgrossvater stammenden Manuskripte ist, schreibt darüber: „Die Korrespondenz mit H. v. Kleist, Novalis, Jean Paul, Fichte, A. v. Humboldt und Raumer ist nicht mehr aufzufinden gewesen; sie warde in der Zeit, wo das Autographensammeln seinen Aufschwung nahm aus den Geschäftspapieren gestohlen." (S. Ungedruckte Briefe an Georg Andreas Reimer, mitgeteilt von G. Hirzel in Deutsche Rundschau, 18. Jahrg., IV. Bd., S. 99.) Diese Nachricht ist, was Fichte betrifft, nicht ganz genau. In der Annahme, dass sich Briefe Fichtes noch im Besitz von Reimers Nachfolger befinden müssten, habe ich mich an diesen gewandt, und Herr Dr. W. de Gruyter als gegenwärtiger Inhaber der Firma Georg Reimer hatte die Freundlichkeit, mir die Abschriften zweier interessanter Briefe Fichtes an Reimer zu ühersenden, mit der Bemerkung, dass im Archiv der Firma ausser diesen noch andere auf Verlagskontrakte bezilgliche Notizen von Fichtes Hand aufbewahrt werden. Die Familie Reimer besitzt dagegen kein Dokument dieser Freundschaft, und konnte auf meine Anfrage nur dieNachricht geben, die ich S.501 Anm. 1 anführe. 
Nachdem der Atheismusstreit für Johann Gottlieb Fichte den Verlust der Professur in Jena zur Folge gehabt hatte, siedelte er nach Berlin über, zuerst, im Juli 1799, allein, einige Monate später mit seiner Familie. Ungefähr zu derselben Zeit war der um vierzehn Jahre jüngere Georg Andreas Reimer, als Leiter der Langeschen Buchhandlung nach Berlin gekommen, der kurz darauf die berühmte, von Hecker 1749 in Anschluss an seine Realschule begründete Realschulbuchhandlung pachtweise übernahm, und durch die Herausgabe von Schleiermachers Monologen einen glänzenden Verlegererfolg erreichte. ${ }^{1}$ )

Auf die Vermittlung Schleiermachers, mit welchem Fichte in der ersten Zeit seines berliner Aufenthaltes in täglichem Verkehr stand, ${ }^{2}$ ) ist wahrscheinlich auch die Bekanntschaft Fichtes mit Reimer zürückzuführen. Es fehlte ihnen übrigens nicht an anderen Annäherungspunkten. Ein eifriger Gönner Reimers war der Buchhändler Sander, ein intimer Freund des als freisinniger katholischer Geistlicher bekzannten Ignaz Aurel Fessler, zu dem Fichte in engen freimaurerischen Beziehungen stand $;^{3}$ ) und auch mit dem Buchhändler Unger, der zu den besten Freunden Fichtes aus dieser Zeit zählte, und in dessen Hause Fichte in einer grossen Gesellschaft den Anfang des neunzehnten Jahrhunderts feierte, ${ }^{4}$ ) war wahrscheinlich Reimer gut bekannt. ${ }^{5}$ )

Es steht jedenfalls fest, dass schon Anfang 1801 ein sehr freundschaftliches Verhältnis zwischen Fichte und Reimer bestand. Das beweist nicht nur unser Billet, sondern auch ein ebenso freundlicher Brief Fichtes an Reimer vom Februar 1801, worin die Bedingungen für die Veröffentlichung von Fichtes „Sonnenklaren

1) Siehe Jonas, Art. Georg Andreas Reimer in der allgemeinen deutschen Biographie, Bd. '27, S. 709-712.

?) S. Fichtes Leben und lit. Briefw., Bd. I, S. 314.

3) S. Allgemeines Handbuch der Freimaurerei ${ }^{3}$, Leipzig 1900, Bd. I, S. 283-4. Fessler scheint überhaupt mit mehreren Buchhändlem auf gutem Fuss gestanden zu haben. Durch seine Vermittlung schloss auch Fichte für. seine „Bestimmung des Menschen“ einen vorteilhaften Verlagsvertrag mit der Voss'schen Buchhandlung ab. S. Fichtes Leben ${ }^{2}$, Bd. I, S. 328.

4) S. Fichtes Leben Bd. I, S. 328.

5) Dafür spricht Reimers le 'haftes Interesse für den von Unger verlegten "Alarcos" von Fr. Schlegel, wovon gleich unten die Rede sein wird, und auch der Umstand, dass später der ganze Bestand von Ungers Verlag an Reimer ïberging, wie mir Herr Dr. de Gruyter mitteilt. 
Bericht an das grössere Publikum über das eigentliche Wesen der neuesten Philosophie" mit grosser Offenheit besprochen werden. ${ }^{1}$ ) Das Buch erschien auch noch in demselben Jahre bei der Realschulbuchhandlung, und war das erste einer Reihe Fischtescher Werke, die Reimer verlegte.

Fichte interessierte sich überhaupt für den Verlag, und in einem Brief von Anfang 1802 bot er Reimer ein Werk eines seiner jenaischen Schüler an. ${ }^{2}$ ) Aus demselben Brief entnehmen wir, dass die Freunde eine Diskussion über den literarischen Wert von Schlegels "Alarcos" gehabt hatten, die schliesslich zur Übereinstimmung beider in einem sehr günstigen Urteil führte.)

1) Das ist einer der beiden Briefe, die im Archiv der Firma Georg Reimer existieren. Fichte verhehlt darin nicht, Geld dringend zu brauchen; es ist übrigens aus der Biographie des Solnes bekannt, dass Fichte in dieser Zeit nur von seiner schriftstellerischen Tätigkeit lebte. Der Brief trägt weder Datum noch Ortsangabe, und nennt auch das Werk nicht, wovon die Rede ist; aus seinem Inhalt lässt sich aber dies alles leicht entnehmen.

2) Das geschieht eben in dem zweiten der genannten Briefe. Das Werk war nach Fichtes Angaben „eine durchgreifende Philosophie der Gesetzgebung". Ich glaube festgestellt zu haben, um welche Schrift es sich handelt. - Fichte nennt in dem Brief den Verfasser "einen der besten Köpfe, die mir während meines Lehramtes in Jena vorgekommen". In seinen „Ideen für dié innere Organisation der Universität Erlangen" (180う-6) spricht Fichte von einem seiner besten Schüler in Jena, einem Juristen, der aber "seine Wissenschaft mit philosophischem Geiste durchdrungen und erfasst hat" und schlägt ihn für eine Professur in Erlangen vor. Dieser Jurist heisst Dresler und hat eine „Organonomie der gesamten Rechtslehre geschrieben, die voll Geist ist" (Fichte, Nachgelassene Werke, Bd. III., S. 291-2). Nun ist im Jahre 1803 bei Reimer in Berlin ein Buch von Karl Dressler erschienen mit dem Titel: „Über das Verhältnis des Rechts zum Gesetze". Man kann also kaum zweifeln, dass dieses Buch das von Fichte empfohlene ist.

3) Das Trauerspiel "Alarcos « von Friedrich Schlegel wurde in Weimar aufgeführt, und nur die Gunst Goethes vermochte es vor einem Misserfolg zu bewahren. Der Druck wurde von A. W. Schlegel bei Unger in Berlin besorgt. Fichte scheint über das Stück, das er vorlesen gehört hatte, ein ungünstiges Urteil ausgesprochen zu haben, worauf Reimer, welcher (vielleicht von Schleiermacher beeinflusst) anderer Ansicht war, ihm das Stück zum lesen gab. In dem Brief nimmt Fichte sein Urteil zurïck, und erklärt den Alarcos für ein „einzig in seiner Art dastehendes Meisterwerk ${ }^{\text {. Man }}$ sehe wie Schleiermacher für den Alarcos begeistert war in A us Schleiermacher's Leben. In Briefen, 1860-63, Bd. I., S. 286; und vgl. Haym, Die romantische Schule?. Berlin 1906, S. 672-3. - Am 18. März 1802 gab Friedrich Schlegel dem Bruder den Auftrag, ein Dedikationsexemplar 
Was aber der Freundschaft dieser beiden besondere Festigkeit verlieh, war ihre demokratische Gesinnung und warme Vaterlandsliebe: dies ist der Punkt, in welchem sich diese durch Wesen und Beschäftigung so verschieden 'gearteten Männer mit gleichem Eifer und gleicher Begeisterung auf gemeinsamen Arbeitsfeld zusammenfanden.

Wir wissen, dass Reimer $\mathrm{zu}^{\circ}$ den ständigen Zuhörern der Privatvorlesungen gehörte, die Fichte in den ersten Jahren seines berliner Aufenthaltes in seiner Wohnung hielt. ${ }^{1}$ ) Von diesen Vorlesungen verlegte Reimer, in einem mit Ungeduld erwarteten Buch, die über „Die Grundzüge des gegenwärtigen Zeitalters", welche im Winter 1804/05 gehalten worden waren, und deren tiefer geschichtsphilosophischer Gehalt den Anfang von Fichtes unmittelbarer Einwirkung auf die politische Erneuerung Deutschlands bezeichnet. ${ }^{2}$ )

Kurz nachher begann der Krieg, und bald lieferte die Schlacht von Jena Berlin den Franzosen aus. Da Fichte nicht in der vom Feinde besetzten Stadt bleiben wollte, zog er in freiwilliger Verbannung nach Königsberg, wohin auch der Hof übersiedelte, fest entschlossen, erșt nach einer gründlichen Änderung der Dinge zurückzukehren. Reimer blieb, aber mit ebenso stolzen Vorsätzen. Als die französischen Militärbehörden von den Berliner Bürgern die Ablieferung sämtlicher Waffen forderten, gab Reimer die seinigen, obwohl er deren viele im Hause hatte, nicht heraus, und soll, nach Fouqué's Erzählung, den warnenden Freunden entgegnet.haben: "Lasst sie suchen bei mir; ich kann ihnen nicht wehren. Und wenn sie was finden, lasst sie mich erschiessen, wenn sie wollen und können. Ich überliefere mich nicht freiwillig, wehrlos in ihre Gewalt; die Wehr bedingt den Mann, kein Mann ohne Wehr.") Auch wurde mancher seiner vom Eroberer verfolgten Freunde (darunter Ernst Moritz Arndt) nicht ohne Gefahr bei ihm beherbergt, wie sein Haus überhaupt zum Sammelplatz aller jener

des Alarcos Fichte zugehen zu lassen (Friedrich Schlegels Briefe an seinen Bruder August Wilhelm, hrsg. v. Walzel, Berlin 1890, S. 492). Der Brief Fichtes wird also ungefähr in dieser Zeit geschrieben worden sein.

1) S. Fichte. Lichtstrahlen aus seinen Werken und Briefen, Leipzig 1863, S. 101.

2) Fichte hatte seit mehreren Jahren nichts veröffentlicht, doch hatte sich der Ruf seiner berliner Vorlesungen verbreitet; daher die Erwartung, wovon hauptsächlich einzelne Briefe Fr. Schlegels an Reimer Zeuguis ablegen. S. Hirzel, Ungedruckte Briefe an G. A. Reimer, S. 101-103. 3) Allg, deutsche Biographie, Bd. 27, S. 710. 
wurde, die sich als Deutsche fühlten und an der Erhebung des Vaterlandes arbeiten wollten. ${ }^{1}$ )

Dass an einem solchen Mann die zurückgelassene Frau Fichtes eine bedeutende Stütze hatte, ist selbstverständlich, und aus zwei Briefen, die Fichte aus Kopenhagen an sie richtete, ist ersichtlich, dass die beiden Familien in steter Beziehung zu einander standen. In diesen Briefen lässt Fichte Reimer den Vorschlag für die Herausgabe eines periodischen Werkes zugehen, welches den Titel „Zur Geschichte des wissenschaftlichen Geistes zu Anfang des 19. Jahrhunderts" tragen sollte, und worin er unter anderm auch seine beiden Dialoge über Patriotismus zu veröffentlichen gedachte. Sein Vorschlag kam aber nicht zur Ausführung und die Dialoge wurden erst in seinen nachgelassenen Werken zum Abdruck gebracht. ${ }^{2}$ )

Nach dem Tilsiter Frieden kehrte Fichte nach Berlin zurück, und im Winter 1807/8 hielt er seine berühmten Reden an die Deutsche Nation. Es ist bekannt, welcher Mut dazugehörte, diese Reden in einer Stadt zu halten, deren Gouverneur ein französischer Marschall war, und in welcher die Stimme des Redners oft genug von französischen Tronmelschlägen übertönt wurde, während sich bekannte Spione im Hörsaal befanden. Fichte war sich auch der Gefährlichkeit seiner Tat vollkommen bewusst. ${ }^{3}$ ) Was man aber gewöhnlich ausser Acht lässt ist, dass dieselbe Verantwortung und dieselbe Gefahr von einem zweiten geteilt wurde, und dieser zweite war Reimer.

Fichte hieit es nämlich für vorsichtig, die Reden gleich während des Vortrages dem Drucke zu übergeben, um etwaige in Umlauf gebrachte Gerüchte über seine Äusserungen durch diesns authentische Zeugnis sofort zum Schweigen bringen zu können. Nun erschienen diese Reden bei der Realschulbuchhandlung, deren allgemein bekannter Besitzer und persönlicher Leiter Reimer war. Zu einer Zeit aber, da die Hinrichtung des Buchhändlers Palm

1) Varnhagen von Ense, Denkwürdigkeiten des eigenen Lebens, Leipzig 1870, II. T., S. 63.

2) Fichtes Leben', I., S. 394 und 397-8; und Fichte, Nachgelassene Werke, III., S. 221-274.

3) Am 2. Jasuar 1808 schrieb er an Beyme: „Ich weiss recht gut, was ich wage, ich weiss, dass ebenso wie Palm ein Blei mich treffen kann. Aber dies ist es nicht was ich fürchte, und für den Zweck, den ich habe,

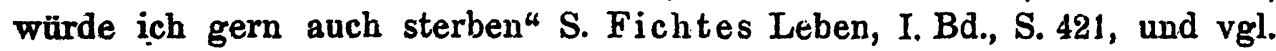
II. Bd., S. 408. 
noch bei allen in frischer Erinnerung stand, und die Franzosen die Stadt noch besetzt hielten, war offenbar die Gefahr für den Verleger eines solchen Buches nicht viel kleiner als für den Verfasser. Sogar die deutschen Zensurbehörden machten aus Angst vor den Franzosen eine Menge Schwierigkeiten, welche Verzögerungen und Ärgernisse aller Art zur Folge hatten, und auch den Verleger schädigten, der sich bei diesem Unternehmen doch gewiss viel weniger von der Hoffnung auf Gewinn als von der Überzeugung leiten liess, für das Wohl seines Vaterlandes $\mathrm{zu}$ arbeiten und eine ernste Bürgerspflicht zu erfüllen. ${ }^{1}$ )

Reimer wurde in der Tat nicht nur Verleger dieser Reden, sondern auch einer der eifrigsten Vorkämpfer der in ihnen verfochtenen Prinzipien; und als 1813 die Stunde des Kampfes und der Befreiung schlug, fand sie Reimer und Fichte wiederum in gleicher Gesinnung vereinigt. Während Fichte vergebens seine Dienste als Feldprediger beim Heere anbot und sich mit den Übungen beim Landsturm und begeisterten politischen Vorlesungen bescheiden musste, trat Reimer, Frau und Kinder sowie alle seine Geschäfte im Stich lassend, als Freiwilliger in das Heer ein; die

1) S. Max Lehmann, Fichtes Reden an die deutsche Nation vor der preussischen Zensur in Preuss. Jahrbücher, 82. Bd., S. 501 bis 515 (1895), auch in seine Histor. Aufsätze, 1911. - Gleich für die erste Rede wurde das Imprimatur verweigert, und da Fichte sich anfangs zu keiner Änderung entschliessen wollte, musste der Druck mit der zweiten Rede begonnen werden. Das Manuskript der dreizehnten Rede ging bei den Zensurbehörden verloren! Dies alles trug natürlich zum Gelingen der Ausgabe nicht bei, und bezüglich des Verlustes der 13. Rede erklärte Reimer den Behörden, dass ihm dieser vertragmässig zu einer Entschädigung von wenigstens 2000 Taler (!), dem Verfasser gegenüber, verpflichtete. Dieser Zwischenfall verursachte vielleicht $z$ wischen Fichte und Reimer eine Verstimmung (s.' die Beilagen zu Lehmanns Aufsatz), die aber jedenfalls nicht von Dauer sein konnte (s. Hirzel, Ungedruckte Briefe an G. A. Reimer, S. 108-9). Fichte mag überhaupt für einen Verleger kein bequemer Verfasser gewesen sein. Dass Reimer allgemein als Verleger der Reden bekannt war, obwohl auf dem Buch nur "Realschulbuchhandlung" stand, beweist auch die Tatsache, dass er in einer offiziellen Mitteilung über den Verlust der dreizehnten Rede ohne weiteres als Verleger genannt wird. - Der die Reden an die deutsche Nation betreffende Verlagskontrakt existiert noch im Archiv der Firma Reimer in Berlin. Seine Veröffentlichung, die mir nicht vergönnt war, wäre in mehreren Hinsichten sehr wünschenswert, und wird hoffentlich nicht allzulange auf sich warten lassen. Merkwürdig ist, dass der Vertrag erst am 3. Mărz 1808 geschlossen wurde, als der Druck schon sehr vorgeschritten war. 
edlen Frauen der beiden Freunde aber zeichneten sich durch aufopferungsvolle Pflege der verwundeten Soldaten aus. ${ }^{1}$ )

Als im Juni 1814 Reimer nach Berlin zurückkehrte, um sich mit seiner in der Zwischenzeit schwergeprüften Familie zu vereinigen ${ }^{2}$ ) und sich wieder seinen Geschäften zu widmen, die er in Kürze zu höchster Blüte brachte, war Fichte bereits seit einigen Monaten tot, so dass er das Andenken des Freundes nur noch mit der Herausgabe seiner letzten politischen Vorlesungen ehren konnte. ${ }^{3}$ ) Das tranrige Geschick des Vaterlandes sollte aber die beiden Namen noch einmal verbinden.

In den Tahren dunkler Reaktion, welche auf die Befreiung folgten, entging Reimer dem Schicksal nicht, das damals die besten Patrioten (selbst die gemässigsten unter ihnen, wie seinen Frennd Schleiermacher) traf, als Demokrat verdächtigt zu werden; während seiner Abwesenheit wurde sein Haus durchsucht, seine Privatpapiere wurden beschlagnahmt. ${ }^{4}$ ) Dieses Schicksal blieb Fichte nur durch den Tcd erspart, $\left.{ }^{5}\right)$ traf aber sein edelstes Buch. Seine Reden an die deutsche Nation wurden von den Mainzer Untersuchungsbehörden als ein „verführerisches, leere Phantome nährendes Buch" bezeichnet, und als eine neue Auflage davon nötig geworden war, wurde zu des damaligen Preussens Schande die Druckerlaubnis in Berlin verweigert. ') So musste Reimer, der 1808 unter den Augen der Franzosen die erste Ausgabe der Reden hatte verbreiten dürfen, 1824 im befreiten Preussen auf die Herausgabe der zweiten verzichten, die dann in Leipzig bei einer andern Firma erschien.

(Deutsch von M artha Ravà.)

1) In der Familie Reimer hat sich die Erinnerung an diese gemeinsame Arbeit der beiden Frauen noch lebhaft erhalten, wie Herr Baurat Conrad Reimer in Berlin-Lichterfelde die Freundlichkelt hatte mir mitzuteilen.

2) Ein Kind Reimers war inzwischen gestorben.

3) J. G. Fichte, Die Staatslehre, Berlin, G. Reimer, 1820. Der politisch bezeichnendste Teil dieser Vorlesungen wurde schon 1815 unter dem Titel „Über den Begriff des wahrhaften Krieges" veröffentlicht.

4) S. Herm. Reimer, Georg Andreas Reimer, Erinnerungen aus seinem Leben insbesondere aus der Zeit der DemagogenVerfolgung, Berlin 1900.

ๆ) \#品te ihn die Kriegsseuche nicht so schnell, so unerwartet dahingerafft, der Friede würde ihn zu seinem ersten Martyrer gemacht haben". So beschliesst mit Recht Kälisch seine Rede zum hundertsten Geburtstag Fichtes. S. Die Fichtefeier der Berliner Mitglieder des Nationalvereins, Berlin 1862, S. 26. II, S. 84 .

9) Fichtes Leben², I, S. $423 \mathrm{Jg}$.; und Varnhagen von Ense a. a. O., 